\title{
Application of the Mode Intermittent Radiation in Fading Channels
}

\author{
Mihail Andrianov ${ }^{1,2}$ and Igor Kiselev ${ }^{3}$ \\ ${ }^{1}$ Institute of Radio-Engineering and Electronics of Russian \\ Academy of Science (Kotelnirov's Institute) \\ ${ }^{2}$ Ltd Research \& Development Company "Digital Solution" \\ ${ }^{3} \mathrm{OAO}$ "Network Technologies" \\ Russia
}

\section{Introduction}

The effectiveness of radio lines which use radio signals from deep fading signal levels can be increased by the use of transmitting devices to adapt to changing the radio channel's parameters. To adapt to the conditions of radio propagation and effects of interfering signals are used the system with: automatic adjustment of transmitter power; automatic variable speed transmission; adaptation in frequency, switching modulation types, etc. To improve noise immunity can be used as the mode of intermittent radiation or intermittent radio communications. Intermittent signal transmission principle provides the highest level of agreement when using this mode in one direction of the double-side radio line.

For a long time, almost until the end of the XX-th century, transmission of radio signals on double-side radio line was carried out on the same principles and with the same energy potential in the forward and reverse directions. Such lines are called symmetric. Currently being developed and are used asymmetric radio lines, in which the transmission of signals in forward and reverse directions is organized on different principles and with different energy potentials. There are a variety of options for the construction of forward and reverse directions.

In constructing the double-sided asymmetric radio lines in either direction can be used by the mode of intermittent radiation. The principal feature of this option is the ability to completely turn off the transmitter during the fading signals and thus reduce the average output power.

In the well-known radio lines of intermittent communication (for example meteor communication), when an intermittent communication is carried out in both directions is not possible to turn off the transmitter. Interruption of communication in both directions leads to the necessity at all times to transmit sounding signals and completely turn the transmitter on the break of communication is not possible. There are other technical difficulties in the organization of bilateral intermittent communication.

More simply a double-side line will created when using intermittent communications in one direction of communication, especially when using technology TDD (Time Divide Duplex). 
In the case of TDD fading signal levels at the receiver inputs at opposite ends of the radio lines are correlated. This allows measuring the signal level at the input of the receiver to estimate the signal level at the other end, and thus dispense with a special back channel communications. In recent decades, great importance is the communication with mobile objects. For such objects, it is often important to minimize the average power consumption of the power supply and increase the duration of their work. From this perspective, the opportunity to switch off the transmitter while fading, and not just to stop the transmission of information signals is an important factor.

Intermittent mode radiation can be used in combination with other ways to improve radio communications. The development of radio engineering has led to what is now practically all the possible ways of improving known and well understood, therefore, appears to be the most significant results to further improve the radio communication systems can be obtained by complexation of different ways. Sometimes there are situations when the use of one method for increasing the efficiency entails reducing the effectiveness of another, but by introducing restrictions on the parameters of signals and separate units of equipment able to maintain the effectiveness of both methods.

It is interesting to consider the use of complex methods diversity of signals and intermittent of radiation. The use of diversity transfer mode reduces the efficiency of intermittent radiation. However, for small number branches of the diversity efficiency of intermittent radiation mode remains significant. There are several options complexation mode of intermittent radiation and diversity signal. In essence, the intermittent mode radiation can be combined with different variants of MIMO (Multiply Input Multiply Output), the use of which is one approach to improving the system of broadcast signals. A number of advances in microelectronics, the possibility of solutions to complex analytical problems have led to many versions of MIMO schemes are implemented in telecommunication systems.

Earlier studies performed immunity intermittent communication concerned only cases receiving single and Rayleigh fading. Similar studies with other statistics of fading weren't made. Study the effectiveness of intermittent connection when its application with the diversity of signals is unknown to authors.

In this chapter the noise immunity of transmission digital communications in fading channels is analyzed in case using the intermittent radiation.

\section{The analysis of noise immunity radio-line at usage mode intermittent of radiation}

\subsection{Error probably of signal reception, use factor of radio-line when usage intermittent radio communication channel with different types of fading}

The main parameter that characterizes the noise immunity of digital communication systems is the probability of error. Because in considered channels of communication signal propagation suffers fading, the probability of error is a random variable, and the quality of communication is estimated by the mean probability of error, which is usually defined as mathematical expectation this random variable. Using the known expression, determining the probability of error for noncoherent reception of orthogonal signals in channels with Gaussian noise and the probability density function of the signal / noise ratio (SNR), was 
found analytic expressions for the dependence of the average probability of error from the mean SNR and the level of threshold's interruption for the most commonly used when describing the fading distribution laws of random variables:

$$
\begin{aligned}
& P_{n c}\left(\gamma_{0}\right)=\frac{1}{2 \cdot \eta\left(\gamma_{0}\right)} \int_{\gamma_{t}}^{\infty} \exp (-\alpha \gamma) \cdot \frac{1}{\gamma_{0}} \cdot \exp \left(-\frac{\gamma}{\gamma_{0}}\right) d \gamma=\frac{1}{2 \cdot \eta\left(\gamma_{0}\right)} \frac{\exp \left[-\alpha \gamma_{t}\left(1+\frac{1}{\alpha \gamma_{0}}\right)\right]}{1+\alpha \gamma_{0}}, \\
& P_{n c}\left(\gamma_{0}\right)=\frac{1}{2 \eta\left(\gamma_{0}\right)} \int_{\gamma_{t}}^{\infty} \exp (-\alpha \gamma) \frac{1}{\gamma_{0}-\gamma_{a}} \cdot \exp \left(-\frac{\gamma+\gamma_{a}}{\gamma_{0}-\gamma_{a}}\right) \cdot I_{0}\left(\frac{2 \cdot \sqrt{\gamma \cdot \gamma_{a}}}{\gamma_{0}-\gamma_{a}}\right) d \gamma, \\
& P_{n c}\left(\gamma_{0}\right)=\frac{1}{4 \eta\left(\gamma_{0}\right) \sqrt{\pi}} \int_{\gamma_{t}}^{\infty} \frac{1}{\gamma} \cdot \frac{1}{\sqrt{\ln \gamma_{0}-\ln \gamma_{0_{-} r e f}}} \exp \left[-\frac{\left(\ln \sqrt{\frac{\gamma}{\gamma_{0_{-} r e f}}}\right)^{2}}{\ln \gamma_{0}-\ln \gamma_{0_{-} r e f}}\right] \cdot e^{-\alpha \gamma} d \gamma, \\
& P_{n c}\left(\gamma_{0}\right)=\frac{1}{2 \eta\left(\gamma_{0}\right)} \frac{1}{\Gamma(m)}\left[\frac{m}{\gamma_{0} \cdot \eta\left(\gamma_{0}\right)}\right]^{m} \int_{\gamma_{t} \eta\left(\gamma_{0}\right)}^{\infty} \gamma^{m-1} \exp \left[-\left(\frac{m}{\gamma_{0} \cdot \eta\left(\gamma_{0}\right)}+\alpha\right) \gamma\right] d \gamma= \\
& =\frac{1}{2} \frac{1}{\Gamma\left(m, \frac{\gamma_{t}}{\gamma_{0}} m\right)}\left[1+\alpha \frac{\gamma_{0}}{m} \frac{\Gamma\left(m, \frac{\gamma_{t}}{\gamma_{0}} m\right)}{\Gamma(m)}\right]^{-m}\left[\Gamma\left[m, m \frac{\gamma_{t}}{\gamma_{0}}+\alpha \gamma_{t} \frac{\Gamma\left(m, \frac{\gamma_{t}}{\gamma_{0}} m\right)}{\Gamma(m)}\right]\right]
\end{aligned}
$$

where analytical expressions (1-4) define mean values probability of error under the laws accordingly Rayleigh, the generalized Rayleigh, lognormal and Nakagami, $m$-parameter of fading and $m>0.5, \alpha$ - the constant coefficient equal 0.5 and 1 for accordingly frequency and phase demodulations, $\gamma, \gamma_{0}, \gamma_{t}-$ accordingly current, average and threshold value SNR, $\eta\left(\gamma_{0}\right)$ - the parameter named use factor (utilization factors or fill factor) of a radio-line, $\Gamma(m)=\int_{0}^{\infty} t^{m-1} e^{-t} d t$ and $\Gamma(m, n)=\int_{n}^{\infty} t^{m-1} e^{-t} d t$ accordingly gamma-function and incomplete gamma-function. Here and below value $\alpha$ will make 1 .

Integral in the formula (4) is tabular, defined by expression

$$
\int_{u}^{\infty} x^{v-1} \exp (-\mu x) d x=\frac{\Gamma(v, \mu \cdot u)}{\mu^{v}} .
$$

Value $\gamma_{a}$ correspond SNR for the regular component of a signal when a signal fading down under laws of the generalized Rayleigh (formula 2). When a signal fading under the lognormal law (formula 3 ) value $r_{0}$ ref. correspond SNR in the nonperturbed environment. 
Random changes of an envelope of a signal of millimeter wave range at a lognormal fading, unlike a fading under Rayleigh, the generalized Rayleigh and Nakagami laws, are caused not by its total interference components, and fluctuation of dielectric transmittivity $\varepsilon$, a wave refraction index $n$ owing to turbulence of troposphere.

Expression $\eta\left(\gamma_{0}\right)$ defines use factor radio-line at intermittent communication (the relation of transmission time of the data to the general time of a communication session) and its lowering corresponds to reduction of spectral efficiency, accordingly for the above-stated types of a fading will make, according to analytical expressions (6-9)

$$
\begin{aligned}
& \eta\left(\gamma_{0}\right)=\int_{\gamma_{t}}^{\infty} \cdot \frac{1}{\gamma_{0}} \cdot \exp \left(-\frac{\gamma}{\gamma_{0}}\right) d \gamma=\exp \left(-\frac{\gamma_{t}}{\gamma_{0}}\right), \\
& \eta\left(\gamma_{0}\right)=\int_{\gamma_{t}}^{\infty} \frac{1}{\gamma_{0}-\gamma_{a}} \cdot \exp \left(-\frac{\gamma+\gamma_{a}}{\gamma_{0}-\gamma_{a}}\right) \cdot I_{0}\left(\frac{2 \cdot \sqrt{\gamma \cdot \gamma_{a}}}{\gamma_{0}-\gamma_{a}}\right) d \gamma, \\
& \eta\left(\gamma_{0}\right)=\int_{\gamma_{t}}^{\infty} \frac{1}{2 \sqrt{\pi}} \cdot \frac{1}{\gamma} \cdot \frac{1}{\sqrt{\ln \gamma_{0}-\ln \gamma_{0_{-} r e f}}} \exp \left[-\frac{\left(\ln \sqrt{\frac{\gamma}{\gamma_{0 \_} r e f}}\right)^{2}}{\ln \gamma_{0}-\ln \gamma_{0_{-} r e f}}\right] d \gamma=\frac{1}{2} \cdot \operatorname{erfc}\left(\frac{1}{2} \cdot \frac{\ln \gamma_{t}-\ln \gamma_{0_{-} r e f}}{\sqrt{\ln \gamma_{0}-\ln \gamma_{0_{-} r e f}}}\right), \\
& \eta\left(\gamma_{0}\right)=\frac{1}{\Gamma(m)}\left(\frac{m}{\gamma_{0}}\right)^{m} \int_{\gamma_{t}}^{\infty} \gamma^{m-1} \exp \left(\frac{\gamma \cdot m}{\gamma_{0}}\right)=\frac{\Gamma\left(m, m \frac{\gamma_{t}}{\gamma_{0}}\right)}{\Gamma(m)},
\end{aligned}
$$

where $\operatorname{erfc}(x)=\frac{2}{\sqrt{\pi}} \int_{x}^{\infty} \exp \left(-t^{2}\right) d t$ - error function complement. Integral in the formula (9) is tabular (5). Analytical expression (6) is a special case of the formula (9) at $m=1$.

Let's accept $\gamma_{t}=k \gamma_{0},(0<k<\infty)$ and value $\mathrm{k}$ is fixed, then the use factor of a radio- line in case of the Rayleigh fading is fixed, doesn't depend from $\gamma_{0}$. In cases of a fading of a signal under laws of the generalized Rayleigh, lognormal and Nakagami it depends accordingly on ratios $k, \gamma_{a}, \gamma_{0} ; k, \gamma_{0_{-} r e f}, \gamma_{0}$ and $k, m$, and in two last cases the value of the specified coefficient will be defined by formulas

$$
\eta\left(\gamma_{0}\right)=\frac{1}{2} \cdot \operatorname{erfc}\left[\frac{1}{2} \cdot\left(\frac{\ln k}{\sqrt{\ln \frac{\gamma_{0}}{\gamma_{0_{-} r e f}}}}+\sqrt{\ln \frac{\gamma_{0}}{\gamma_{0_{-} r e f}}}\right)\right] ; \eta(m)=\frac{\Gamma(m, k m)}{\Gamma(m)} .
$$

Analyzing dependences of probability of errors on mean value SNR, it is necessary to consider that at constant instantaneous power of the transmitter at intermittent 
communication energy of bit of a signal decreases proportionally to use factor of a radioline. Therefore for, the comparative analysis of noise immunity at intermittent communication and without it, it is expedient in the first case at calculation of probability of error accordingly to reduce level of a threshold and an average of value SNR and that is showed in analytical expression (4). In the absence of it, for correct comparing of the specified probabilities of errors, it is necessary to admit that instantaneous power transmitters at intermittent communication comparing communication without interruption is increased in inverse proportion to use factor of radio-line that is illustrated in formulas (1-3).

Application algorithm of intermittent communication reduces probabilities of errors in the channel with a fading and, especially with growth of mean value SNR $\left(\gamma_{0}\right)$ at some lowering of spectral efficiency (use factor of a radio-line). For example, for the Rayleigh channel (1) at $\gamma_{t}=\gamma_{0} ; \eta\left(\gamma_{0}\right)=1 / \mathrm{e},(6)$ therefore

$$
P_{n c}\left(\gamma_{0}\right)=\frac{e}{2} \int_{\gamma_{0}}^{\infty} \exp (-\alpha \gamma) \cdot \frac{1}{\gamma_{0}} \cdot \exp \left(-\frac{\gamma}{\gamma_{0}}\right) d \gamma=\frac{1}{2} \frac{\exp \left(-\alpha \gamma_{0}\right)}{1+\alpha \gamma_{0}}=\frac{1}{2} \frac{1}{1+\alpha \gamma_{0}} \cdot \exp \left(-\alpha \gamma_{0}\right) \text {. }
$$

Thus, application of intermittent communication with a threshold equal $\gamma_{0}$ reduces probability of error incoherent reception in comparing probability of errors in the Rayleigh channel without interruption exponentially, in $\exp \alpha \gamma_{0}$ time.

\subsection{Comparing of energetic effectiveness usage of intermittent communication and noise immunity coding}

The comparative analysis of energetic efficiency of the radio-line using intermittent communication in comparison with a radio-line, using the noise immunity code for the channel with a fading of Nakagami is carried out. Variation of coefficient $k$ we will select for $m=1.4$ such value of a threshold $\left(\gamma_{t}=k \gamma_{0}\right)$ that for them $\eta(9)$ it was equal 0.5 .

Comparing of effectiveness of communication is expedient for carrying out on energetic coding gain and energetic gain of intermittent communication at the given probabilities of errors. Energetic coding gain for intermittent communication $(m=1.4 ; \eta=0.5)$ and the convolutional code of speed 0.5 are presented to tab. 1. At realization of intermittent communication, as well as transmission of an encoded signal, energy of the bit (average power) at the fixed instantaneous power of the transmitter will decrease twice, the width of spectra of both signals thus will double, and spectral efficiency accordingly twice will decrease.

\begin{tabular}{|l|c|c|c|c|}
\hline The given probabilities of error & $10^{-3}$ & $10^{-4}$ & $10^{-5}$ & $10^{-6}$ \\
\hline Energetic gain of coding $(\mathrm{dB})$ & 4 & 5,1 & 5,7 & 6 \\
\hline $\begin{array}{l}\text { Energetic gain of intermittent } \\
\text { communication }(\mathrm{dB})\end{array}$ & 11,3 & 16,1 & 22,7 & 28,9 \\
\hline
\end{tabular}

Table 1. 
The data presented to tab. 1 show that intermittent communication, is more effective comparing convolutional code usage (at equal values of use factor of radio-line and speed of coding). Besides, in the channel with a fading for possibility of realization of decoding of the convolutional code, originating burst errors necessary to convert in single-error. Normally it is fulfilled by operations of interleaving/de-interleaving which are implemented with additional hardware expenses that causes additional signal delays. At deeper fading of a signal when $0.5<m<1$ energetic gain in application of intermittent communication will increase.

\section{Complexation of intermittent communication and diversity reception}

\subsection{Substantiation of expediency of complexation of intermittent communication and diversity reception of signals}

It is known that at use of diversity reception fading in the channel becomes less deep, due it increases the noise immunity of signal reception. At complexation intermittent communication with diversity reception, gain comparing to diversity reception without intermittent communication will be less than that of an intermittent connection, comparatively the continuous reception in a single. And this gain decreases with magnification of number of branches of diversity as with growth of the last depth of a fading decreases. However usage of intermittent communication at restricted number of branches of the diversity, normally put into practice, leads to noticeable magnification of noise immunity. Besides, application of diversity reception at intermittent communication increases use factor of radio-line that also should raise noise immunity of reception and spectral efficiency of data transfer.

Value $\eta_{M}$, named use factor of radio-line, essentially influences noise immunity of reception of signals. At complexation of intermittent communication and diversity reception in communication paths for calculation probability of error reception it is necessary to define use factor of radio-line at various number of branches of diversity $(M)$ depending on $\gamma_{0}$.

\subsection{Analysis of noise immunity at complexation of intermittent communication and diversity reception with a combination branches of diversity on algorithm of an auto select}

It is known that the probability density function at $M$ - the multiple diversity reception with an ideal auto select at an independent homogeneous fading can be defined from expression

$$
W_{M}(\gamma)=\frac{d\left(\int_{0}^{\gamma} f_{\gamma} d \gamma\right)^{M}}{d \gamma},
$$

where $M$ is number of branches of diversity, $f_{\gamma}$ - probability density SNR at various types of a fading.

At complexation of intermittent communication and diversity reception with a combination of branches of diversity on algorithm of an auto select, use factor of radio-line at various number of branches of diversity $(M)$ depending on $\gamma_{0}$ are defined according to expression 


$$
\eta_{M}\left(\gamma_{0}\right)=\int_{\gamma_{t}}^{\infty} W_{M}(\gamma) d \gamma=\int_{\gamma_{t}}^{\infty} \frac{d\left(\int_{0}^{\gamma} f_{\gamma} d \gamma\right)^{M}}{d \gamma} d \gamma .
$$

Use factor of radio-line at complexation of intermittent communication and diversity reception with a combination of branches of diversity on algorithm of an auto select at a signal fading under accordingly Rayleigh (12), the generalized Rayleigh (13), lognormal (14) and Nakagami (15) laws will be defined according to (11) expressions

$$
\begin{aligned}
& \eta_{M}\left(\gamma_{0}\right)=\int_{\gamma_{t}}^{\infty} \frac{d\left[1-\exp \left(-\frac{\gamma}{\gamma_{0}}\right)\right]^{M}}{d \gamma} d \gamma=1-\left[1-\exp \left(-\frac{\gamma_{t}}{\gamma_{0}}\right)\right]^{M}, \\
& \eta_{M}\left(\gamma_{0}\right)=\int_{\gamma_{t}}^{\infty} \frac{d\left[\int_{0}^{\gamma} \frac{1}{\gamma_{0}-\gamma_{a}} \cdot \exp \left(-\frac{\gamma+\gamma_{a}}{\gamma_{0}-\gamma_{a}}\right) \cdot I_{0}\left(\frac{2 \sqrt{\gamma_{a} \cdot \gamma}}{\gamma_{0}-\gamma_{a}}\right) d \gamma\right]^{M}}{d \gamma} d \gamma, \\
& \eta_{M}\left(\gamma_{0}\right)=\int_{\gamma_{t}}^{\infty} \frac{d\left[\frac{1}{2}+\frac{1}{2} \operatorname{erf}\left(\frac{1}{2} \cdot \frac{\ln \gamma-\ln \gamma_{0_{\_} r e f}}{\sqrt{\ln \gamma_{0}-\ln \gamma_{0_{-} r e f}}}\right)\right]^{M}}{d \gamma} d \gamma= \\
& =1-\left[\frac{1}{2}+\frac{1}{2} \cdot \operatorname{erf}\left(\frac{1}{2} \cdot \frac{\ln \gamma_{t}-\ln \gamma_{0 \_r e f}}{\sqrt{\ln \gamma_{0}-\ln \gamma_{0 \_} r e f}}\right)\right]^{M} \\
& \eta_{M}\left(\gamma_{0}\right)=\frac{M}{\Gamma(m)}\left(\frac{m}{\gamma_{0}}\right)^{m} \int_{\gamma_{t}}^{\infty}\left[1-\frac{\Gamma\left(m, \frac{\gamma}{\gamma_{0}} m\right)}{\Gamma(m)}\right]^{M-1} \exp \left(-\frac{\gamma}{\gamma_{0}} m\right) \gamma^{m-1} d \gamma= \\
& =1-\left[\frac{\Gamma(m)-\Gamma\left(m, \frac{\gamma_{t}}{\gamma_{0}} m\right)}{\Gamma(m)}\right]^{M}
\end{aligned}
$$

where $\operatorname{erf}(x)=\frac{2}{\sqrt{\pi}} \int_{0}^{x} \exp \left(-t^{2}\right) d t$ error function.

Analytical expression (12) is a special case of the formula (15) at $m$, equal 1. Dependence of use factor of radio-line on number of branches of diversity for a fading under the law of Nakagami, at $\gamma_{t}=\gamma_{0}$ and $m$ equal 0.7 , under the formula (15), is presented on fig. 1. 


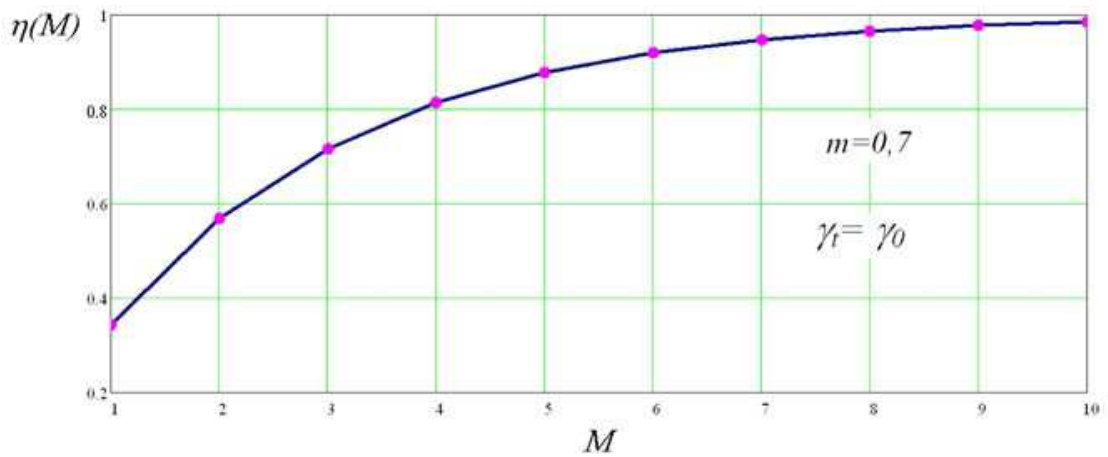

Fig. 1. Dependence of use factor of radio-line from $M$ for a fading under the law of Nakagami, at $\gamma_{t}=\gamma_{0}$ and $m$ equal 0.7 .

Similarly, methods of calculation for a single reception and intermittent communication is defined depending on the average error probability of noncoherent reception in the complexation intermittent links and diversity reception of the average SNR $\left(\gamma_{0}\right)$ for the cases of the fading signal differential binary phase shift key (D-BPSK), respectively, under the laws of the Rayleigh (16) [], the generalized Rayleigh (17) [], log-normal (18) [] and Nakagami (19)

$$
\begin{aligned}
& P_{M}\left(\gamma_{0}\right)=\frac{1}{2 \eta_{M}} \cdot \int_{\gamma_{t} \cdot \eta_{M}\left(\gamma_{0}\right)}^{\infty} \frac{d\left[1-\exp \left(-\frac{\gamma}{\gamma_{0}}\right)\right]^{M}}{d \gamma} \cdot \exp (-\alpha \gamma) d \gamma= \\
& \frac{1}{2 \eta_{M}\left(\gamma_{0}\right)} \cdot M \cdot \exp \left[-\alpha \gamma_{t} \cdot \eta_{M}\left(\gamma_{0}\right)\right] \cdot\left[\sum_{k=1}^{M} C_{M-1}^{k-1} \cdot \frac{(-1)^{k-1} \cdot \exp \left(-\frac{k \gamma_{t}}{\gamma_{0}}\right)}{\alpha \gamma_{0} \cdot \eta_{M}\left(\gamma_{0}\right)+k}\right] \\
& P_{M}\left(\gamma_{0}\right)=\frac{1}{2 \eta_{M}\left(\gamma_{0}\right)} \int_{\gamma_{t}}^{\infty} \exp (-\alpha \gamma) \frac{d\left[\int_{0}^{\gamma} \frac{1}{\gamma_{0}-\gamma_{a}} \cdot \exp \left(-\frac{\gamma+\gamma_{a}}{\gamma_{0}-\gamma_{a}}\right) \cdot I_{0}\left(\frac{2 \sqrt{\gamma_{a} \cdot \gamma}}{\gamma_{0}-\gamma_{a}}\right) d \gamma\right]^{M}}{d \gamma} d \gamma \text {, } \\
& P_{M}\left(\gamma_{0}\right)=\frac{1}{\eta_{M}} \int_{\gamma_{t} \eta_{M}}^{\infty} M \cdot\left\{\frac{\left[\frac{1}{2}+\frac{1}{2} \cdot \operatorname{erf}\left(\frac{1}{2} \cdot \frac{\ln \gamma-\ln \gamma_{0_{-} \text {ref }} \eta_{M}}{\sqrt{\ln \gamma_{0}-\ln \gamma_{0_{-} r e f}}}\right)\right]^{M-1}}{2 \sqrt{\pi}} \cdot \frac{\exp \left[-\frac{1}{4} \frac{\left(\ln \gamma-\ln \gamma_{0_{-} r e f} \eta_{M}\right)^{2}}{\ln \gamma_{0}-\ln \gamma_{0_{-} r e f}}\right]}{\gamma \cdot \sqrt{\ln \gamma_{0}-\ln \gamma_{0_{-} r e f}}}\right\} \frac{\exp (-\alpha \gamma)}{2} d \gamma^{\prime} \\
& P_{M}\left(\gamma_{0}\right)=\frac{M}{2 \eta_{M}\left(\gamma_{0}\right)}\left[\frac{m}{\gamma_{0} \eta_{M}\left(\gamma_{0}\right)}\right]^{m} \frac{1}{\Gamma(m)} \int_{\gamma_{t} \eta_{M}\left(\gamma_{0}\right)}^{\infty}\left\{1-\frac{\Gamma\left[m, \frac{m \gamma}{\gamma_{0} \eta_{M}\left(\gamma_{0}\right)}\right]}{\Gamma(m)}\right\}^{M-1} \quad \gamma^{m-1} \exp \left\{-\left[\frac{m}{\gamma_{0} \eta_{M}\left(\gamma_{0}\right)}+\alpha\right] \gamma\right\} d \gamma \cdot
\end{aligned}
$$


Dependences of probability of errors at a signal fading under the law of Nakagami, and complexation of intermittent communication and diversity reception with a combination of branches of diversity on algorithm of an auto select, at $\gamma_{t}=\gamma_{0}$ and at $m$ equal 0.7 in comparison with continuous diversity reception are shown in Fig. 2.

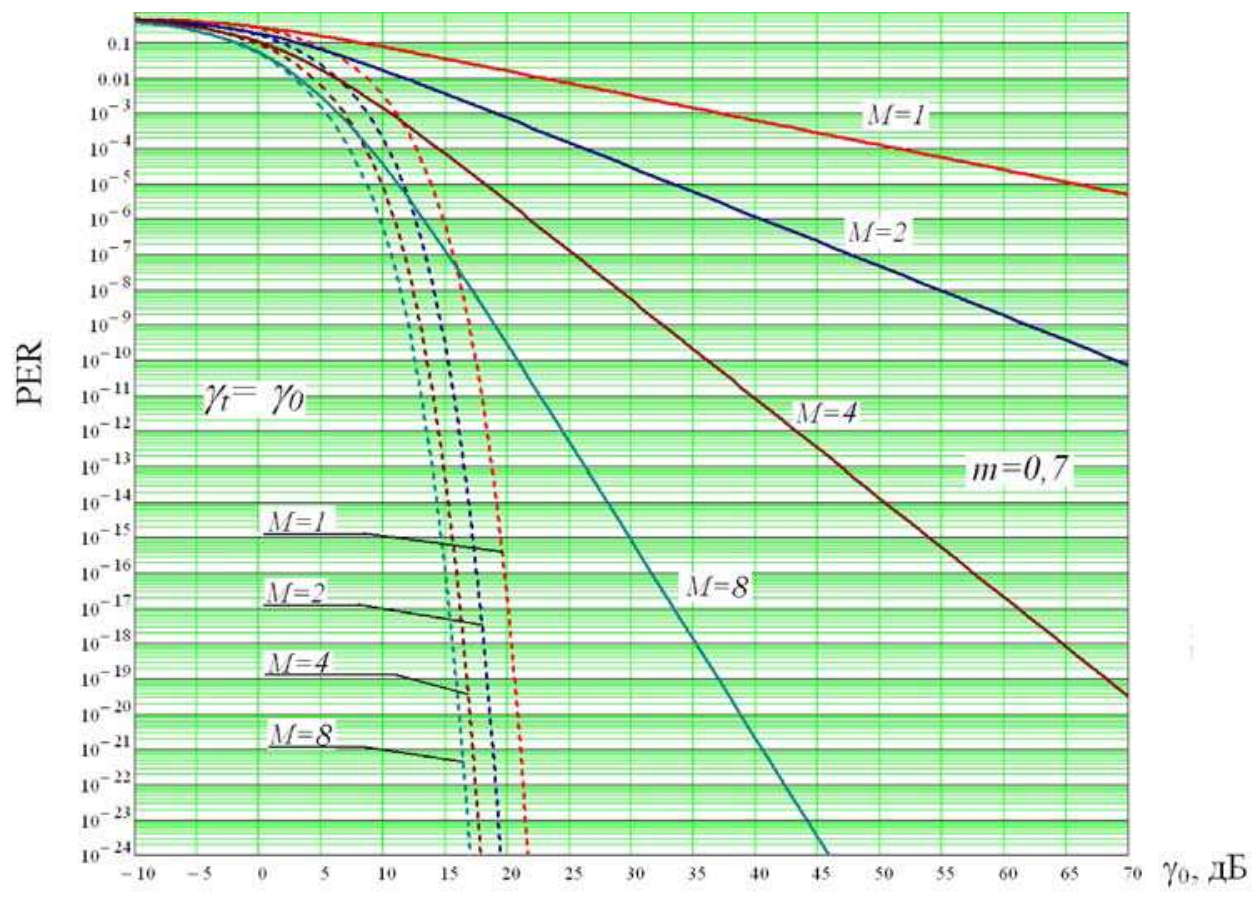

Fig. 2. Probabilities of errors at complexation of intermittent communication and diversity reception, with a combination of branches of diversity on algorithm of an auto select for a signal fading under the law of Nakagami at $\gamma_{t}=\gamma_{0}$ and at $m$ equal 0.7 in comparison with continuous diversity by reception (continuous curves) at various number of branches of diversity $(M)$.

Dependence of probability of an error at a lognormal fading of a signal (18) is obtained for a non stationary case, when the dispersion of the lognormal process defined by a ratio at fixed $\gamma_{0 \_}$ref. isn't a constant

$$
2 \sigma_{\chi}^{2}=\ln \left\langle X^{2}\right\rangle=\ln \gamma_{0}-\ln \gamma_{0_{-} r e f .}=\ln \frac{\gamma_{0}}{\gamma_{0_{-} r e f .}}
$$

In practice, at calculation of noise immunity of communication lines the dispersion of lognormal process normally is the fixed value. For this case it is expedient to define analytical expressions of probability density SNR (21), use factor of radio-line $(22,23)$ and probabilities of error reception of incoherent signal differential D-BPSK (24) in the conditions of a lognormal fading at complexation of intermittent communication and 
diversity reception with a combination branches of diversity on algorithm of an auto select. The diagrams illustrating specified dependences are presented accordingly on fig. 3, 4, 5. Analytical expression of probability density SNR for the specified type of a fading is defined by a method of symbolic mathematical modeling in the environment of MathCAD 14

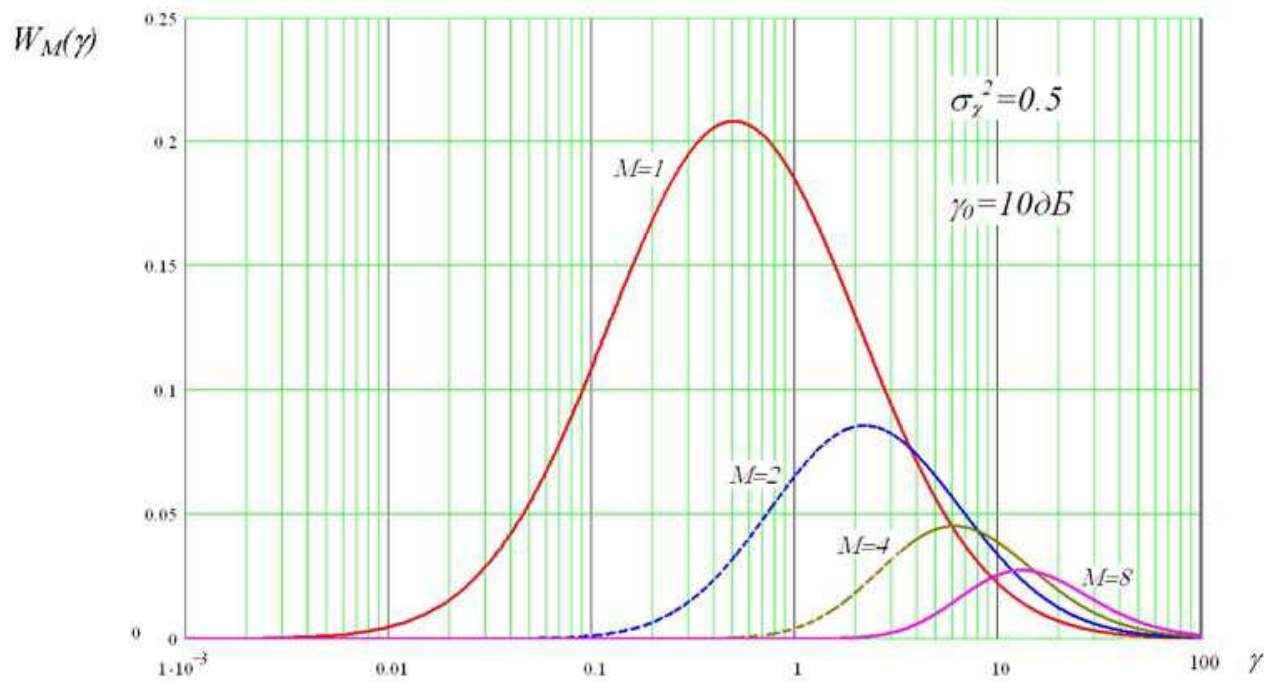

Fig. 3. Probability densities SNR diversity reception of signals in the channel with a lognormal fading at combination of branches of diversity on algorithm of an auto select, at the fixed dispersion $\left(\sigma_{\chi}^{2}\right)$ and mean value SNR $\left(\gamma_{0}\right)$.

$$
\begin{gathered}
W_{M}(\gamma)=\frac{M}{2} \cdot \frac{1}{\sqrt{2 \pi \sigma_{\chi}^{2}} \gamma} \cdot\left[\frac{1}{2}+\frac{1}{2} \cdot \operatorname{erf}\left(\frac{\sqrt{2}}{2} \cdot \frac{\ln \sqrt{\frac{\gamma}{\gamma_{0}}}+\sigma_{\chi}^{2}}{\sigma_{\chi}}\right)\right]^{M-1} \cdot \exp \left[-\frac{\left(\ln \sqrt{\frac{\gamma}{\gamma_{0}}}+\sigma_{\chi}^{2}\right)^{2}}{2 \sigma_{\chi}^{2}}\right]= \\
=\frac{M}{2} \cdot \frac{1}{\sqrt{2 \pi \sigma_{\chi}^{2}} \gamma} \cdot\left[\left(\frac{\ln \sqrt{\frac{\gamma}{\gamma_{0}}}+\sigma_{\chi}^{2}}{\sigma_{\chi}}\right)\right]^{M-1} \cdot \exp \left[-\frac{\left(\ln \sqrt{\frac{\gamma}{\gamma_{0}}}+\sigma_{\chi}^{2}\right)^{2}}{2 \sigma_{\chi}^{2}}\right],
\end{gathered}
$$

the use factor of radio-line will thus be defined under the formula

$$
\eta_{M}\left(\gamma_{0}\right)=\int_{\gamma_{t}}^{\infty} W_{M}(\gamma) d \gamma=1-\left[\frac{1}{2}+\frac{1}{2} \operatorname{erf}\left(\frac{1}{\sqrt{2}} \frac{\ln \sqrt{\frac{\gamma_{t}}{\gamma_{0}}}+\sigma_{\chi}{ }^{2}}{\sigma_{\chi}}\right]\right]^{M}=1-\left[\Phi\left(\frac{\ln \sqrt{\frac{\gamma_{t}}{\gamma_{0}}}+\sigma_{\chi}{ }^{2}}{\sigma_{\chi}}\right)\right]^{M} .
$$


At $\gamma_{t}=\gamma_{0}$ the use factor of radio-line depends only on number branches of diversity, fig. 4

$$
\eta_{M}=1-\left[\frac{1}{2}+\frac{1}{2} \operatorname{erf}\left(\frac{\sigma_{\chi}}{\sqrt{2}}\right)\right]^{M}=1-\left[\Phi\left(\sigma_{\chi}\right)\right]^{M},
$$

where $\Phi(\mathrm{x})$ is normal cumulative distribution function.

The probability of error reception for signal D-BPSK will be defined thus according to expression

$$
P_{M}\left(\gamma_{0}\right)=\frac{1}{\eta_{M}} \int_{\gamma_{0} \eta_{M}}^{\infty} W_{M}(\gamma) \cdot \frac{\exp (-\alpha \gamma)}{2} d \gamma .
$$

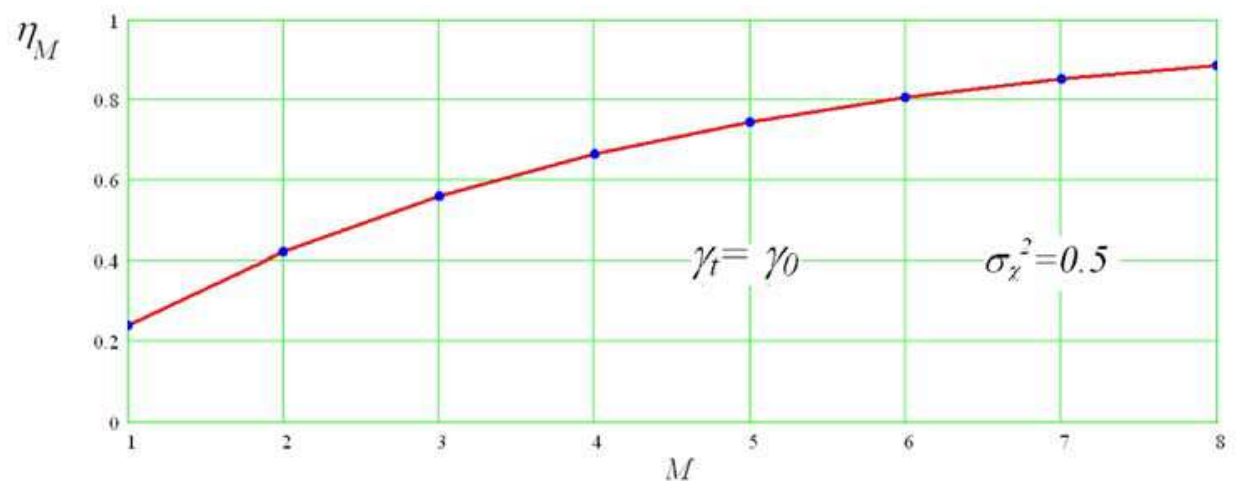

Fig. 4. Dependence of use factor of radio-line from number branches of diversity $(M)$ at $\gamma_{t}=\gamma_{0}$ and the fixed dispersion $\left(\sigma_{\chi}^{2}\right)$.

In the fading signal under the laws of Nakagami and lognormal use factor of the radio line increases, respectively, with increasing parameter of fading $(m)$ and lowering variance $\left(\left(\sigma_{\chi}^{2}\right)\right.$. The probabilities of errors are reduced under these conditions.

\subsection{The noise immunity analysis at complexation of intermittent communication with diversity reception and optimal addition of branches diversity}

Let's consider a variant of diversity reception of signal D-BPSK when combination of branches of diversity is carried out by a method of optimal addition for which the equality defined by expression is valid

$$
P_{M}\left(\gamma_{\Sigma}\right)=\frac{1}{2} \exp \left(-\sum_{i=1}^{M} \gamma_{i}\right)
$$

where value SNR of optimally added signal $\left(\gamma_{\Sigma}\right)$ will be to equally arithmetical total SNR of all of $i$ channels.

It is possible to show that the average probability of an error after optimal addition will be defined by the formula 


$$
P_{M}=\frac{1}{2} \int_{0}^{\infty} \cdots \int_{0}^{\infty} \exp \left(-\sum_{i=1}^{M} \gamma_{i}\right) W_{M}\left(\gamma_{1} \cdots \gamma_{M}\right) d \gamma_{1} \cdots d \gamma_{M}
$$

where $W_{M}\left(\gamma_{1} \cdots \gamma_{M}\right)$ - is a combined $M$-dimensional density of probabilities.

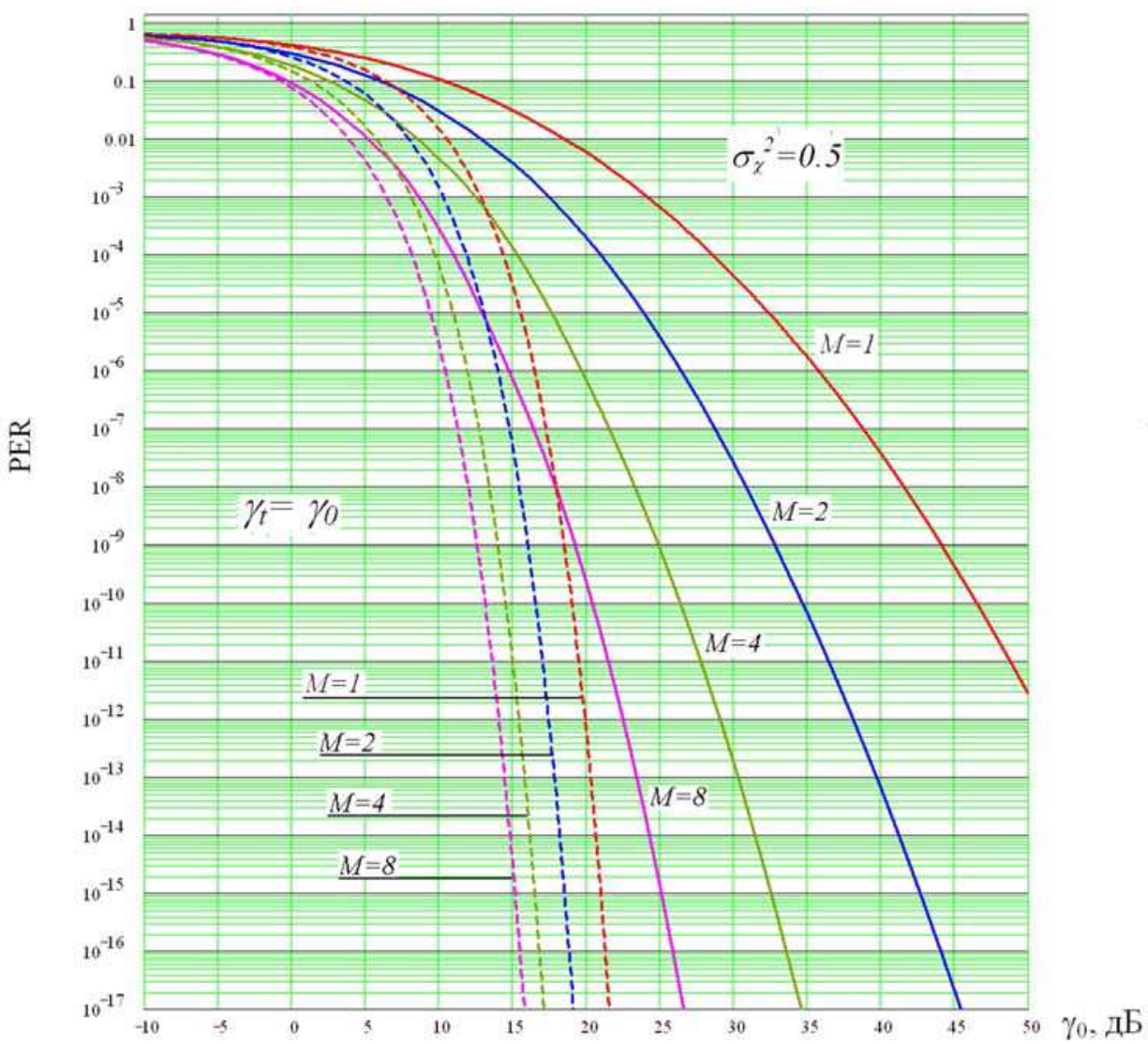

Fig. 5. Probabilities of errors of diversity reception of signals in the channel with a lognormal fading at combination of branches of diversity on algorithm of an auto select (continuous curves) and probabilities of errors at complexation of intermittent communication and diversity reception at combination of branches of diversity on algorithm of an auto select for $\gamma_{t}=\gamma_{0}, \sigma_{\chi}^{2}=0.5$ and $M=1,2,4,8$.

Using the fact that the independent fading diversity branches in the combined probability density is the product of probability densities in separate branches of reception according to the analytical expression

$$
P_{M}=\frac{1}{2} \prod_{i=1}^{M} \int_{0}^{\infty} \exp \left(-\gamma_{i}\right) W_{i}\left(\gamma_{i}\right) d \gamma_{i}
$$


and with homogeneous channels in branches of diversity the probability of error reception on an output of the demodulator signal D-BPSK after operation of optimal addition will be described by the formula

$$
P_{M}=\frac{1}{2}\left[\int_{0}^{\infty} \exp (-\gamma) W(\gamma) d \gamma\right]^{M} .
$$

Formulas $(27,28)$ are valid at any distribution laws.

The average probability of error at complexation of the intermittent communication which are carried out in branches of diversity with the subsequent optimal addition and incoherent demodulation of signal D-BPSK at a fading of an envelope of a signal under laws Rayleigh and Nakagami will be defined accordingly by expressions $(29,30)$

$$
\begin{gathered}
P_{M}\left(\gamma_{0}\right)=\frac{1}{2}\left[\frac{1}{\eta\left(\gamma_{0}\right)} \int_{\gamma_{t} \eta\left(\gamma_{0}\right)}^{\infty} \exp (-\alpha \gamma) \cdot \frac{1}{\gamma_{0}} \cdot \exp \left(-\frac{\gamma}{\gamma_{0}}\right) d \gamma\right]^{M}= \\
=\frac{1}{2}\left[\frac{1}{1+\gamma_{0} \eta\left(\gamma_{0}\right)}\right]^{M} \exp \left[-M \gamma_{t} \eta\left(\gamma_{0}\right)\right] \\
P_{M}\left(\gamma_{0}\right)=\frac{1}{2}\left\{\frac{1}{\eta\left(\gamma_{0}\right)} \frac{1}{\Gamma(m)}\left[\frac{m}{\gamma_{0} \cdot \eta\left(\gamma_{0}\right)}\right]^{m} \int_{\gamma_{t} \eta\left(\gamma_{0}\right)}^{\infty} \gamma^{m-1} \exp \left[-\left(\frac{m}{\gamma_{0} \cdot \eta\left(\gamma_{0}\right)}+\alpha\right) \gamma\right] d \gamma\right\}^{M}= \\
=\frac{1}{2}\left\{\frac{1}{\Gamma\left(m, m \frac{\gamma_{t}}{\gamma_{0}}\right)} \frac{\Gamma\left[m \frac{\gamma_{t}}{\gamma_{0}}+\alpha \gamma_{t} \eta\left(\gamma_{0}\right)\right.}{\left[1+\alpha \frac{\gamma_{0}}{m} \eta\left(\gamma_{0}\right)\right]^{m}}\right\}^{M}
\end{gathered}
$$

where $\eta\left(\gamma_{0}\right)$ - the use factor of radio-line in a separate branch of diversity, is defined for the specified types of a fading accordingly expressions $(6,9)$, thus level of a threshold of interruption in various branches of diversity is identical. Analytical expression (29) is a special case of the formula (30) at $m$ equal 1.

The probability of presence of a signal in diversity branches, under condition of their homogeneity and independence, is defined binomial by the distribution law, therefore the use factor of radio-line on an output of the circuit of optimal addition will be defined by expression (31)

$$
\eta_{M}\left(\gamma_{0}\right)=1-\left[1-\eta\left(\gamma_{0}\right)\right]^{M}
$$

The diagrams illustrating dependence of probability of an error from mean value SNR at various number of branches of diversity, according to analytical expression (30), at the complexation of intermittent communication, considered above, and diversity reception for a fading of an envelope of a signal under the law of Nakagami, at $\gamma_{t}=\gamma_{0}$ and $m$ equal 0.6 are presented on fig. 6 . 
At application of intermittent communication after performance of operation of optimal addition it is more convenient to define probability density SNR on an output of the circuit of optimal addition by a method of characteristic functions, and then on the obtained probability density to calculate use factor of radio-line and probability of error.

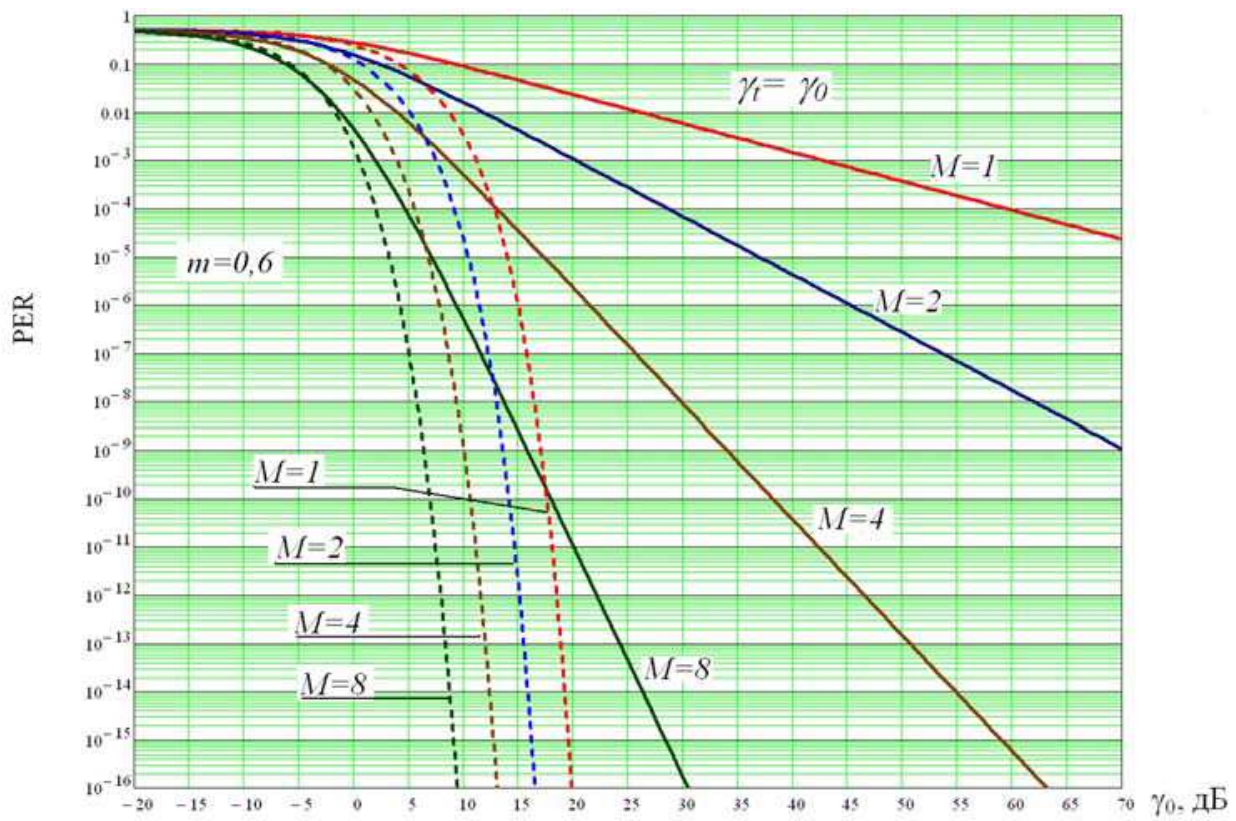

Fig. 6. Probabilities of errors at diversity reception (continuous curves) and probabilities of errors at complexation of intermittent communication carried out in branches of diversity with their subsequent optimal addition, at $M$ equal $1,2,4$ and $8, m$ equal 0,6 and $\gamma_{t}=\gamma_{0}$.

Characteristic function from probability density SNR at a fading an envelope of a signal under the law of Nakagami will be defined by expression

$$
S(v)=\int_{0}^{\infty} f(\gamma) \exp (i v \gamma) d \gamma=\frac{m^{m}}{\Gamma(m)} \frac{1}{\gamma_{0}{ }^{m}} \int_{0}^{\infty} \gamma^{m-1} \exp \left[-\left(\frac{m}{\gamma_{0}}-i v\right) \gamma\right] d \gamma .
$$

Integral in the formula (32) tabular, according to expression

$$
\int_{0}^{\infty} x^{\nu-1} \exp (-\mu x) d x=\frac{\Gamma(v)}{\mu^{v}}
$$

therefore characteristic function will be defined by the formula

$$
S(v)=\frac{m^{m}}{\gamma_{0}^{m}} \frac{1}{\left(\frac{m}{\gamma_{0}}-i v\right)^{m}} .
$$


Probability density SNR in the channel with diversity reception at combination of independent homogeneous channels on algorithm of optimal addition will be defined by inverse transformation from characteristic function in raise to power of number branches of diversity

$$
f_{M}(\gamma)=\int_{-\infty}^{\infty} \frac{1}{2 \pi}[S(v)]^{M} \exp (-i v \gamma) d v=\frac{1}{2 \pi} \frac{m^{m M}}{\gamma_{0}^{m}} \int_{-\infty}^{\infty} \frac{\exp (-i v \gamma)}{\left(\frac{m}{\gamma_{0}}-i v\right)^{m M}} d v
$$

Integral in the formula (35) tabular, defined by to expression

$$
\int_{-\infty}^{\infty}(\beta-i x)^{-v} \exp (-i p x) d x=\frac{2 \pi \cdot p^{v-1} \exp (-\beta p)}{\Gamma(v)},
$$

therefore final expression of probability density SNR at optimal addition branches of diversity will be defined as

$$
f_{M}(\gamma)=\frac{m^{m M}}{\Gamma(m M)} \frac{\gamma^{m M-1}}{\gamma_{0}^{m M}} \exp \left(-\frac{\gamma}{\gamma_{0}} m\right) .
$$

On fig. 7 probability density SNR is presented at optimal addition of branches of diversity, at $m$ equal $0.6 ; 1.4$ and 7 in the absence of the diversity, doubled and quadrupled diversity receptions.

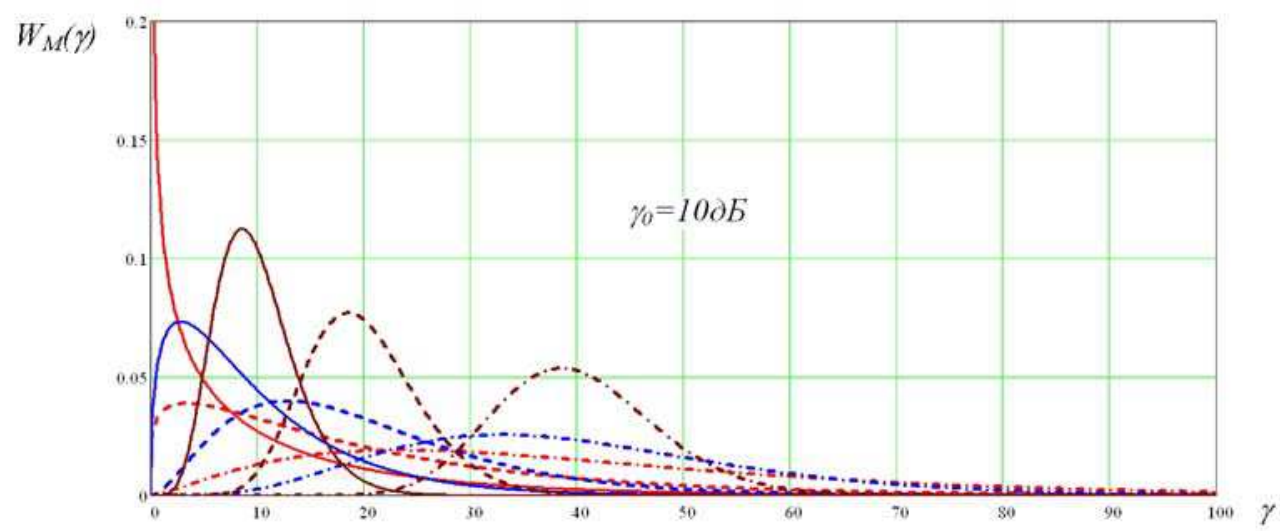

Fig. 7. Probability densities SNR at optimal addition of branches of diversity, at $m$ equal $0.6 ; 1.4$ and 7, accordingly red, dark blue and brown curves, in the absence of diversity; doubled and quadrupled diversity receptions, accordingly continuous, dashed and dash-dotted curves.

Use factor of radio-line, at complexation of diversity reception with optimal addition of branches of diversity and the intermittent communication which are carried out after that addition, in channels with a fading under laws Rayleigh and Nakagami are described accordingly by formulas $(38,39)$ 


$$
\begin{gathered}
\eta_{M}\left(\gamma_{0}\right)=\left(\frac{1}{\gamma_{0}}\right)^{M} \frac{1}{(M-1) !} \int_{\gamma_{t}}^{\infty} \gamma^{M-1} \exp \left(-\frac{\gamma}{\gamma_{0}}\right)=\frac{\Gamma\left(M, \frac{\gamma_{t}}{\gamma_{0}}\right)}{\Gamma(M)}, \\
\eta_{M}\left(\gamma_{0}\right)=\left(\frac{m}{\gamma_{0}}\right)^{m M} \frac{1}{\Gamma(m M)} \int_{\gamma_{t}}^{\infty} \gamma^{m M-1} \exp \left(-\frac{\gamma}{\gamma_{0}} m\right)=\frac{\Gamma\left(m M, \frac{\gamma_{t}}{\gamma_{0}} m\right)}{\Gamma(m M)} .
\end{gathered}
$$

Integrals in expressions (38) and (39) are tabular (5). Analytical expression (38) is a special case of the formula (39) at $m$, equal 1.

Dependences probability of error at incoherent reception of a signal at complexation of diversity reception with optimal addition of branches of diversity and the intermittent communication, which are carried out after that addition, from mean value SNR $\left(\gamma_{0}\right)$, under formulas $(40,41)$ for cases of a fading of signal D-BPSK under accordingly laws Rayleigh and Nakagami will be defined by averaging of probability of errors in Gaussian noise according to the specified fading at values SNR above the given threshold level $\left(\gamma_{t}\right)$

$$
\begin{aligned}
P_{M}\left(\gamma_{0}\right) & =\frac{1}{2 \eta_{M}\left(\gamma_{0}\right)} \frac{1}{\gamma_{0}^{M}} \frac{1}{(M-1) !} \int_{\gamma_{t}}^{\infty} \gamma^{M-1} \exp \left[-\left(\frac{1}{\gamma_{0}}+\alpha\right) \gamma\right]=\frac{1}{2}\left(\frac{1}{1+\alpha \gamma_{0}}\right)^{M} \frac{\Gamma\left(M, \frac{\gamma_{t}}{\gamma_{0}}+\alpha \gamma_{t}\right)}{\Gamma\left(M, \frac{\gamma_{t}}{\gamma_{0}}\right)}, \\
& P_{M}\left(\gamma_{0}\right)=\frac{1}{2 \eta_{M}\left(\gamma_{0}\right)}\left[\frac{m}{\gamma_{0} \eta_{M}\left(\gamma_{0}\right)}\right]^{m M} \frac{1}{\Gamma(m M)} \int_{\gamma_{t} \eta\left(\gamma_{0}\right)}^{\infty} \gamma^{m M-1} \exp \left\{-\left[\frac{m}{\gamma_{0} \eta\left(\gamma_{0}\right)}+\alpha\right] \gamma\right\}= \\
= & \frac{1}{2}\left[1+\alpha \frac{\gamma_{0}}{m} \frac{\Gamma\left(m M, \frac{\gamma_{t}}{\gamma_{0}} m\right)}{\Gamma(m M)}\right]^{-m M} \frac{\Gamma\left[m M, m \frac{\gamma_{t}}{\gamma_{0}}+\alpha \gamma_{t} \frac{\Gamma\left(m M, \frac{\gamma_{t}}{\gamma_{0}} m\right)}{\Gamma(m M)}\right]}{\Gamma\left(m M, \frac{\gamma_{t}}{\gamma_{0}} m\right)}
\end{aligned}
$$

The diagrams illustrating dependence of probability of error from mean value SNR at complexation of diversity reception with optimal addition of branches of diversity and intermittent communication, carried out after that additions, for a fading of an envelope of a signal under the law of Nakagami, at $\gamma_{t}=\gamma_{0}$ and $m$ equal 0.6 is presented on fig. 8.

Dependences use factor of radio-line from number branches of diversity (M) at complexation of diversity reception with optimal addition branches of diversity and intermittent communication, which are carried out after that addition, and use factor of radio-line at complexation of intermittent communication, which carried out in branches of diversity with their subsequent optimal addition, is presented on fig. 9.

The use factor of radio-line at complexation of diversity reception with optimal addition of branches of diversity and the intermittent communication which are carried out after that addition, increases from magnification of branches of diversity $(M)$ faster, than use factor of radio-line at communication of diversity reception and the intermittent communication which 


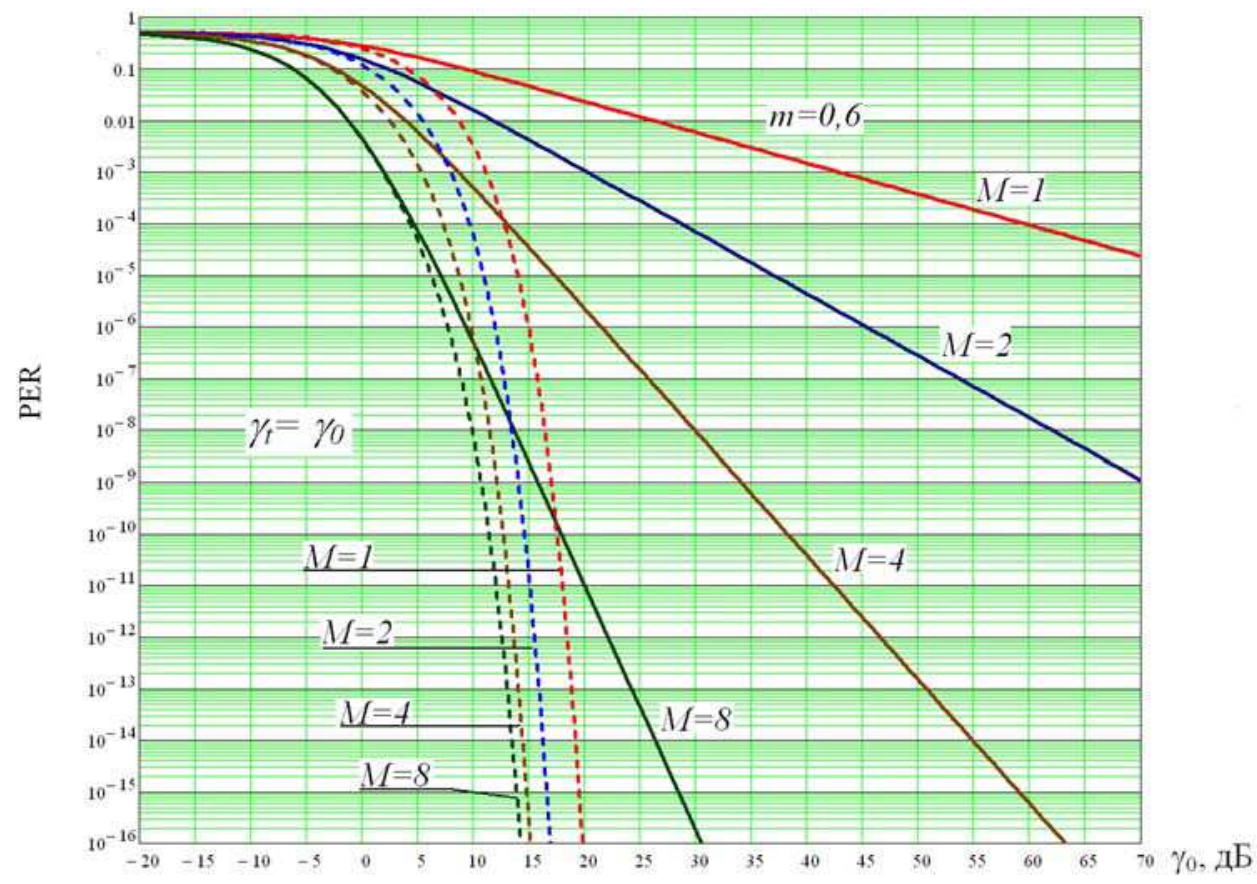

Fig. 8. Probabilities of errors at diversity reception (continuous curves) and probabilities of errors at complexation of diversity reception with optimal addition branches of diversity and the intermittent communication which are carried out after that addition, at $M$ equal $1,2,4$ and $8, m$ equal 0.6 and $\gamma_{t}=\gamma_{0}$.

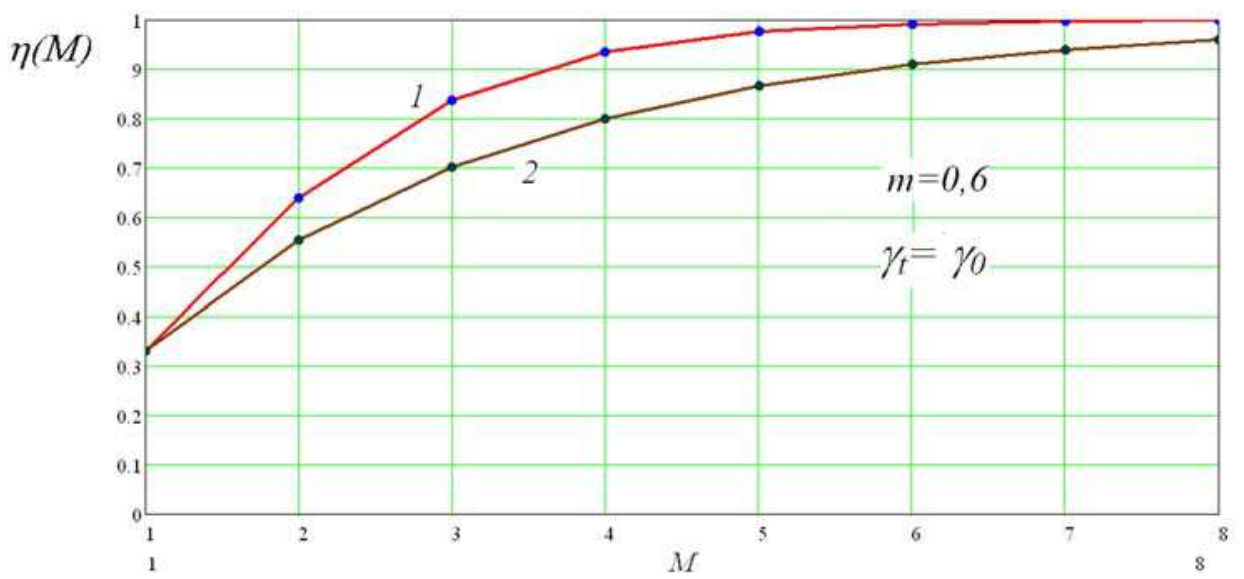

Fig. 9. Dependences of use factor of radio-line from number branches of diversity at $m$ equal 0.6 and $\gamma_{t}=\gamma_{0}$, at complexation of intermittent communication and diversity reception when interruption is carried out on an output of the circuit of optimal addition (a line 1) and when interruption is carried out in diversity branches, with their subsequent optimal addition (a line 2). 
are carried out in branches of diversity with their subsequent optimal addition (fig. 9). Values last are commensurable with use factor of radio-line at complexation of intermittent communication and diversity reception and combine of branches of diversity on algorithm of an auto select (fig. 1), even at smaller value $m$. Accordingly, spectral efficiency thus increases.

Probabilities of errors at complexation of diversity reception and the intermittent communication, which are carried out in branches of diversity with their subsequent optimal addition, decrease with magnification of mean value SNR faster probabilities of errors at complexation of diversity reception with optimal addition branches of diversity and intermittent communication, which are carried out after that addition.

Unlike complexation of intermittent communication and diversity reception with combination branches of diversity on algorithm of an auto select when the receiver selects a peak signal from all branches of diversity, at optimal addition the receiver should accept a signal from all branches for the subsequent handling. For this purpose it is necessary to use some antennas and to provide orthogonality of transmittable signals.

\section{Complexation some methods adaptation of radio-lines}

Let's consider a combination of an intermittent of radiation with constant average power and transmitter's speed automatic adjustment. We will suppose that information transfer rate changes depending on signal level in such a manner that SNR on a receiving device input remains constant. In this case at signal level changes under the Rayleigh law distribution density of probability of the random variable which is transmission rate will be defined by expression

$$
W(\omega)=\frac{r}{\omega_{0}} \exp \left(-\frac{\omega r}{\omega_{0}}\right)
$$

where $\omega$ is information transfer rate, $\omega_{0}$ is a constant for the given system value, $r$ - fixed value SNR

Average rate of information transfer is defined as mathematical expectation of value $\omega$

$$
V_{c p}=\int_{0}^{\infty} \omega \frac{r}{\omega_{0}} \exp \left(-\frac{\omega r}{\omega_{0}}\right) d \omega=\frac{\omega_{0}}{r} .
$$

Median value of speed will be equal

$$
V_{M}=\ln 2 \frac{\omega_{0}}{r}
$$

Let's enter designation $\omega_{t}$ meaning threshold value of the instant transmission rate at which the transmitter is shut down. We will find a number of the elementary signals which are transferred at speed exceeding threshold value. For this purpose we will consider integral

$$
N=\int_{\omega_{t}}^{\infty} \omega \frac{r}{\omega_{0}} \exp \left(-\frac{\omega r}{\omega_{0}}\right) d \omega=\frac{\omega_{0}}{r} \exp \left(-\frac{\omega_{t} r}{\omega_{0}}\right)\left(\frac{r}{\omega_{0}} \omega_{t}+1\right) .
$$


Having divided it into value of average rate from (43) we will obtain a share of characters transferred at excess of threshold value $\omega_{t}$

$$
\frac{N}{V_{c p}}=\exp \left(-\frac{\omega_{t} r}{\omega_{0}}\right)\left(\frac{r}{\omega_{0}} \omega_{t}+1\right)
$$

To transfer all characters which would be transferred without transmitter shut-down it is necessary to increase average rate which we will find, having divided (43) on (46)

$$
V_{c p}^{\prime}=\frac{\omega_{0}}{r} \frac{\omega_{0}}{r \omega_{t}+\omega_{0}} \exp \frac{\omega_{t} r}{\omega_{0}}
$$

The use factor of radio-line in this case is equal

$$
\eta\left(\omega_{t}\right)=\exp \left(-\frac{\omega_{t} r}{\omega_{0}}\right)
$$

From ratios (46) and (48) follows, that at the same level $r$, speed can be increased in comparison with a transmitter's speed automatic adjustment by value

$$
\Delta\left(\omega_{t}\right)=1+\frac{r \omega_{t}}{\omega_{0}}=1+\frac{\omega_{t}}{V_{c p}}
$$

Possible gain at various values of threshold level of speed are presented in table 2

\begin{tabular}{|l|c|c|c|c|c|}
\hline Threshold value of speed & $\omega_{0} / r$ & $\ln 2 \omega_{0} / r$ & $2 \omega_{0} / r$ & $3 \omega_{0} / r$ & $4 \omega_{0} / r$ \\
\hline Power gain & 2 & $\ln 2+1$ & 3 & 4 & 5 \\
\hline
\end{tabular}

Table 2.

\section{Conclusion}

The carried out analysis shows that in channels with random parameters (channels of systems of mobile communication, channels of tropospheric and ionospheric radio propagation, etc.) when the admissible time delay of signals exceeds duration of time intervals in which the relation a signal/noise becomes inadmissible small, application of principles of intermittent communication allows to obtain the considerable gain in noise immunity. The magnification of gain is connected to lowering of spectral efficiency and magnification of delay period of signals.

For saving of average rate of information transfer it is necessary to increase the instant speed and by that to expand occupied frequency band under a condition when in the modern communication systems basic is the requirement of saving of the frequency resource.

The analysis usage complexation of intermittent communication and diversity reception allows not only to lower probability of error an accepted signal, but also sharply to raise use factor of radio-line (spectral efficiency), especially at complexation of diversity reception 
with the intermittent communication which is carried out on an output of the circuit of optimal addition. The magnification of spectral efficiency is very significant owing to limitation of a resource of an electromagnetic spectrum.

The analytical ratios obtained in the given chapter, show, what gain can be implemented at usage methods of intermittent communication in various situations at the various statistics fading amplitudes of signals. These ratios allow calculating also probabilities of error at a fading of signals under laws of Rayleigh, Rice (generalized Rayleigh), Nakagami, and also lognormal laws at incoherent and coherent receptions.

In cases of possible applications for the mode of intermittent radiation (i.e., turn off the transmitter at low signal levels), the most complete communication channel can be used in a combination of variable speed and intermittent modes of communication. Potential possibilities usage methods of intermittent communication especially in a combination to other known methods of increase of noise immunity are considerable.

One of main limitation of method of intermittent communication is necessity of the considerable time delays of signals. In connection with electronics achievements this lack has the lesser value. Mastering of more and more high-frequency ranges of radio-waves leads to possibility to reduce delay period in equipment of these ranges (speed of a fading above). In connection with the marked circumstances it is possible to assume that interest to usage of methods of intermittent communication will increase.

By this time research of systems from intermittent communication is led insufficiently full. The authors suggest investigating these systems under the influence of fading interference from other radiation sources (for example, in the case Rayleigh fading as a useful signal and noise). Researches influence correlations of a fading signal in reception branches are necessary. As different variants of complex application of intermittent communication and diversity of signals (circuits MIMO) are possible, it is supposed to conduct similar researches for other variants of combined usage of intermittent communication and diversity of signals, for example at usage of different levels of thresholds at interruption's realization in different branches of diversity. Usage of intermittent communication when the signal envelope suffers simultaneously some types of a fading is of interest. For example, at propagation of signal in millimeter range through troposphere, a fading of its envelope is carried out simultaneously under laws lognormal and Nakagami. Probability density fading envelope of signal as the special case of a fading discussed (when the signal envelope fluctuates simultaneously under laws Rayleigh and lognormal and under laws Rice and lognormal) has been obtained accordingly Suzuki and Pätzold in 1977 and 1998.

\section{References}

Abramowitz M., Stegun A. (1964). Handbook of mathematical functions with Formulars, Graphs, and Mathematical Tables, National Bureau of standards applied mathematics series-55, Issued June 1964, ISBN 0-486-61272-4

Andrianov M.N., Bumagin A.V., Kalashnikov K.S. \& Sisoev I. Yu. (2011) Increase the noise immunity of data transmission over digital communication channels under Nakagami fading conditions, Measurement Techniques, Vol.54, No. 3, 2011, pp. 57-62, ISSN 1573-8906 
Andrianov M.N., Bumagin A.V., Kalashnikov K.S., Kiselev I.G. \& Sisoev I. Yu. (2011) High performance suboptimal method of transmission and reception of signals in channels with Nakagami fading, 13 th International Conference "Digital Signal Processing and its applications", Moscow, 2011, pp. 14-17, (in Russian) ISSBN 978-5-905278-01-3

Andrianov M.N., Bumagin A.V., Kiselev I.G., Rutkevich A.V., Steshenko V.B., Shishkin G.V. (2010) The analysis and synthesis of discontinuous diversity communication for increase of efficiency of transmission of the discrete messages, $12^{\text {th }}$ International Conference "Digital Signal Processing and its applications", Moscow, 2010, pp. 82-85, (in Russian) ISSBN 978-5-904602-07-9

Andrianov M.N., Bumagin A.V., Gondar A.V., Kalashnikov K.S., Prudnikov A.A. \& Steshenko V.B. (2010). A method of increase the noise immunity and spectral efficiency of data communication channels under condition of generalized Rayleigh fading, Measurement Techniques, Vol.53, No. 8, 2010, pp. 61-65, ISSN 1573-8906

Andrianov M.N. (2009). Features interrupted communication of data transmission through channel under condition of lognormal fading, Metrology, No.5, 2009, pp.35-43, (in Russian), ISSN 0132-4713.

Andrianov M.N. (2009). Rise noise-immunity of mobile communication in lognormal fading by interconnecting interrupt communication with diversity receives, Electromagnetic waves and electronic systems, Vol. 14, No. 8, 2009 (in Russia), pp.11-17 ISSN1560-4128

Andrianov M.N. and Kiselev I.G. (2008). About increase of interference immunity of transmission of the discrete messages in channels with a fading, 10th International Conference "Digital Signal Processing and its applications", Moscow, 2008, pp. 82-85, (in Russian)

Andrianov M. N. and Kiselev I.G. (2007). Probability of error in channels with random parameters when complexing intermittent transmission with diversity reception, Mobile. System, No. 5, 2007, pp. 44-47 (in Russian) ISSN 1729-438X

Andronov I.S. and Fink L.M. (1971). Transmission of the discrete messages on parallel channels, Moscow, Soviet radio, 1971 (in Russian)

Asad Mehmood \& Abbas Mohammed (2010). Characterisation and Channel Modelling for Satellite Communication Systems, InTech, pp. 133-151, ISBN 978-953-307-135-0 , Rijeka, Croatia

Banket V. A. and Dorofeev V. M. (1988). Digital Methods in Satellite Communications, Radio i Svyaz, Moscow (1988) (in Russian) ISBN 5-256-00075-6

Gradshein I.S., Rizhik I.M. (1963). Tables of Integral, Moscow, The main publishing house of the physical and mathematical literature, 1963 (in Russian), ISBN 0-12-294757-6

Kiselev I.G. and Ustyantzev S. V. (2009) Probability of an error in Rayleigh noisy channel at usage of interrupted signal transmission, Mobile Telecommunications, No 1, 2009, pp. 34-36 (in Russian), ISSN 1562-4293

Kiselev I.G. and Andrianov M. N. (2007). A method of increasing the noise protection of digital data transmission in channels with fading, Mobile System, No. 4, 2007, pp. 13-16 (in Russian) ISSN 1729-438X

Klovsky D.D. (1976). Processing of time-space signals (in information transfer channels), Moscow, Svyas, 1976 (in Russian)

Lee W. (1982) Mobile communication engineering, Mc Graw-Hill, 1982, ISBN 0070370397

Proakis J.G. (1995). Digital Communication, Mc Graw-Hill, ISBN 9007-051726-6. 
Rytov S.M., Kravtsov J.A. and Tatarskiy V.I. (1978). Introduction in statistical radio physics, Part II. Random fields, Moscow, Science, 1978 (in Russian)

Shakhtarin B. I., Andrianov M. N., \& Andrianov I. M. (2009). Application of Discontinuous Communications in Channels with Random Parameters to Transmit Narrowband Signals and Signals with Orthogonal Frequency-Division Multiplexing, Journal of Communications Technology and Electronics, 2009, Vol. 54, No. 10, pp. 1173-1180, ISSN 1064-2269

Shloma A.M., Bakulin M.G., Kreidelin V.B., Shumov A.P. (2008) New algorithms of formation and handling signals in mobile communication systems, Moscow, Gorachaya LiniyaTelecom, 2008 ISBN 978-5-9912-0061-5 (in Russian)

Sklar B. (2001). Digital Communication, Fundamental and Application, Second Edition, Prentice Hall PTR, ISBN 13-084788-7

Zuko A.G. (1963). Noise immunity and efficiency system of communications, Moscow, Svyas, 1963 (in Russian) 


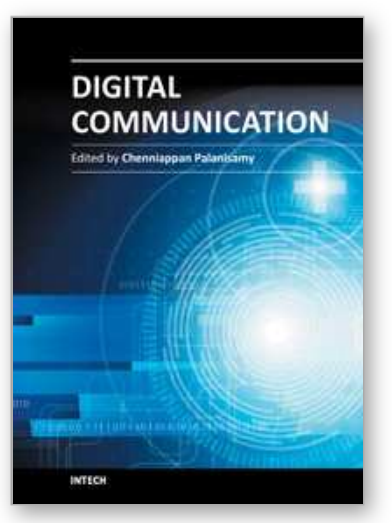

\author{
Digital Communication \\ Edited by Prof. C Palanisamy
}

ISBN 978-953-51-0215-1

Hard cover, 208 pages

Publisher InTech

Published online 07, March, 2012

Published in print edition March, 2012

All marketing is digital and everyone should have a digital strategy. Everything is going mobile. "The world has never been more social" is the recent talk in the community. Digital Communication is the key enabler of that. Digital information tends to be far more resistant to transmit and interpret errors than information symbolized in an analog medium. This accounts for the clarity of digitally-encoded telephone connections, compact audio disks, and much of the enthusiasm in the engineering community for digital communications technology. A contemporary and comprehensive coverage of the field of digital communication, this book explores modern digital communication techniques. The purpose of this book is to extend and update the knowledge of the reader in the dynamically changing field of digital communication.

\title{
How to reference
}

In order to correctly reference this scholarly work, feel free to copy and paste the following:

Mihail Andrianov and Igor Kiselev (2012). Application of the Mode Intermittent Radiation in Fading Channels, Digital Communication, Prof. C Palanisamy (Ed.), ISBN: 978-953-51-0215-1, InTech, Available from: http://www.intechopen.com/books/digital-communication/application-of-the-mode-intermittent-radiation-infading-channels

\section{INTECH}

open science | open minds

\section{InTech Europe}

University Campus STeP Ri Slavka Krautzeka 83/A 51000 Rijeka, Croatia Phone: +385 (51) 770447 Fax: +385 (51) 686166 www.intechopen.com

\section{InTech China}

Unit 405, Office Block, Hotel Equatorial Shanghai No.65, Yan An Road (West), Shanghai, 200040, China 中国上海市延安西路65号上海国际贵都大饭店办公楼405单元 Phone: +86-21-62489820

Fax: +86-21-62489821 
(C) 2012 The Author(s). Licensee IntechOpen. This is an open access article distributed under the terms of the Creative Commons Attribution 3.0 License, which permits unrestricted use, distribution, and reproduction in any medium, provided the original work is properly cited. 